\title{
New genetic testing technologies: Advantages and limitations
}

\author{
A Krause, $\mathrm{MB} \mathrm{BCh}, \mathrm{PhD}$ \\ Division of Human Genetics, National Health Laboratory Service and School of Pathology, Faculty of Health Sciences, \\ University of the Witwatersrand, Johannesburg, South Africa
}

Corresponding author: A Krause (amanda.krause@nhls.ac.za)

Genetic testing has been available for many years. Recently, the potential for increased and more precise diagnostic testing has expanded, with the introduction of next-generation sequencing technologies. Testing is complex and requires the experience of medical geneticists and genetic counsellors to assist in selecting the most appropriate test for a patient, as well as in interpreting the large data sets that arise from such testing. The most appropriate test for a patient may require analysis of only one base of DNA, or one gene, one panel, a whole exome or a whole genome. The interpretation of positive findings is challenging and requires careful distinction between benign and pathogenic variants. Variants of uncertain significance occur; unexpected findings are not infrequent and require careful interpretation and management. The paucity of data on normal variation poses specific challenges on the African continent.

S Afr Med J 2019;109(4):207-209. DOI:10.7196/SAMJ.2019.v109i4.13990

Genetic testing, at some level, has been around since at least the early 1900s. By definition, it involves the analysis of human DNA, RNA, chromosomes, proteins and certain metabolites to detect heritable disease-related genotypes, mutations, phenotypes or karyotypes for clinical reasons. Recent technological advances, particularly nextgeneration sequencing (NGS), have resulted in rapidly reduced costs per base of DNA analysed, with much higher levels of resolution, enhancing the availability and scope of genetic testing, especially for single-gene disorders. These tests offer patients and doctors new and improved opportunities for definitive diagnoses and improved management, while putting an end to long diagnostic odysseys.

It can be complex to choose the correct test for a patient, and the choices can be confusing and overwhelming. Scope for misinterpretation and inappropriate testing is also higher. Although laboratory 'wet' test technology is being increasingly automated, the challenges of analysis, storage and interpretation of large amounts of data are considerable and require highly trained scientists and bioinformaticists. They need to work with genetic counsellors and medical geneticists - in this way the information is accurately interpreted and its clinical relevance is assessed.

The fundamental reasons for performing genetic testing remain unchanged. Patients and doctors require a definitive diagnosis for optimum management. Furthermore, individuals and families have major concerns regarding risks of occurrence and recurrence of genetic disorders. These requirements necessitate appropriate and accurate genetic testing, facilitated by individuals trained in medical genetics and genetic counselling, with detailed knowledge of clinical genetic assessment of patients, choice of tests and interpretation of results.

Testing by DNA analysis offers advantages over testing of other metabolites, as DNA remains unchanged from conception to death, and is typically the same in all tissues. Other metabolites may only be measurable once a disease manifests. Thus, DNA analysis can be performed definitively, once-off and at any stage of life and provides the same results. This necessitates extremely high-level laboratory practice, such that errors are minimised.

\section{Indications for genetic testing}

There are a number of broad indications for genetic testing:

- Diagnostic testing can confirm or rule out a known or suspected genetic disorder in a symptomatic individual.

- Predictive or presymptomatic testing can be offered to asymptomatic individuals to refine their risk of developing a condition later in life.

- Carrier testing identifies individuals who carry autosomal or $\mathrm{X}$-linked recessive mutations, and who may be at risk of having children with a genetic disorder.

- Prenatal or pre-implantation testing is performed prior to or during a pregnancy to assess the health status of a fetus or embryo.

\section{The changing face of genetic testing}

Genetic testing by DNA analysis has been available for an increasing number of single-gene disorders since the mid-1980s. These tests were specifically directed at a single gene or part of a gene. They tested for common mutations or family-specific mutations, or sequenced a relatively small amount of DNA to identify unknown mutations. Typically, no more than 2 - 3 genes would have been analysed simultaneously, owing to the relatively high costs and time to do the analysis. However, results had a high certainty of a predictable result, and it was relatively easy to compare laboratories and quality of results.

Currently, new NGS technologies offer an increasing ability to make accurate diagnoses for single-gene disorders, as multiple genetic analyses can be performed simultaneously. Testing is thus less hypothesis driven. Patients who have rare mutations have improved chances of obtaining diagnoses, complex genetic interactions are being increasingly recognised and new disease-associated genes are being identified. This ultimately translates into improved understanding of genetic causation of disease and more accurate individual and familyrisk assessment. Testing remains relatively expensive, although the cost per base of DNA sequenced has reduced dramatically. The required equipment is expensive to run and maintain. NGS data also remain challenging to interpret owing to the size and complexity 
of the genome and the technical errors that are introduced during sample preparation, sequencing and analysis. These errors can be somewhat mitigated by the use of reference standards for validation and quality control. However, such standards and filtering may also increase the proportion of false-negative results, which represent missed opportunities for diagnosis. Certain sequencing artifacts and short-read misalignment cannot easily be overcome. Furthermore, it is difficult to compare the quality of sequencing offered by different laboratories, as many of these metrics are not standardly provided.

\section{How is a genetic test chosen?}

Testing should prioritise the patient's needs. Currently, from a diagnostic point of view, an older single-gene test may in many cases still provide the fastest and most definitive result, with the least ambiguity. More extensive testing is not necessarily better. Genetic testing should not be performed in the absence of good clinical phenotyping, as correlation of genetic and clinical data is key to interpretation.

The choice of the most appropriate test for a patient can be somewhat overwhelming, as the technologies, test designs, sequence coverage (how much of a gene(s) of interest is sequenced) and depth of coverage (related to accuracy) may differ and not be easily accessible. As each test level offers more analysis, the temptation is to choose a test with more data rather than one with less, in the hope of a higher likelihood of a positive result. This is certainly not always the case. The data sets are extremely large, and the analysis is extremely complex and time-consuming.

There are three broad categories of NGS tests available, each adding a successively larger data load for arguably diminishing diagnostic return:

- Gene-panel testing can be helpful in scenarios where the patient's clinical features are relatively distinct and multiple genes are known to cause a similar phenotype in different individuals. In such cases, a so-called panel of selected genes can be analysed simultaneously. Panels may contain only a few genes or several hundred genes. Such gene-panel tests have a high diagnostic yield if carefully selected and appropriate patients with suggestive clinical features are tested. The panels tend to provide results with high clinical certainty and little ambiguity, e.g. muscular dystrophy, familial cancer or epilepsy gene panels.

- Whole-exome sequencing (WES) includes the analysis of sequence of the entire coding region of the human genome ( $\sim 50$ million DNA bases), with no analysis of non-coding DNA. In this analysis, 1 $2 \%$ of the genome is analysed, with the advantage that $\sim 85 \%$ of pathogenic mutations are located in exons, with a predicted high diagnostic yield. Such analysis may be required when a patient's phenotype is relatively poorly defined or a new syndrome is suspected. However, interpretation can be very challenging and should not be undertaken without consultation with experts in genetics.

- Whole-genome sequencing (WGS) covers all 3000 million bases of human DNA. This analysis obviously generates a massive dataset and a very large number of variants, which would need to be analysed. It has the potential to identify genetic mutations outside the coding regions (e.g. in introns), or where large structural rearrangements are responsible, but is largely a research technique that currently has few clinical indications, particularly as our knowledge of the role of non-coding DNA is rather limited.

It is also important to always consider the implications of a negative test. Despite the highest quality testing, patients with features highly suggestive of a genetic condition may test negative and thus a negative test does not exclude a genetic cause. The reasons for negative tests are not always clear, but may be a combination of missed mutations due to, among other factors, technical issues (parts of the genome do not get sequenced owing to their complex structure), large rearrangements and deletions that are difficult to detect with current NGS techniques, misinterpretation and epigenetic modifications of DNA (where the sequence is unchanged). There may also be mutations in a regulatory region or regions currently not thought to have functional significance.

Chromosomal microarray analysis is an important genetic test that is now considered the gold standard for detecting chromosomal abnormalities. It has increased the identification and resolution of large chromosomal deletions, duplications and rearrangements, typically involving a number of genes. It is often the first test indicated in individuals with developmental delay and other congenital abnormalities. NGS techniques are currently poor at detecting such abnormalities and therefore microarray analysis is often performed prior to NGS or as a complementary test to an NGS-based test, particularly if a large (non-single gene) abnormality is suspected.

\section{Interpretation of genetic tests}

A large number of genetic variants are likely to be identified in any analysis. We are currently in a phase where our technological capabilities outstrip our knowledge and interpretive capabilities. A proportion of variants analysed in any test would be benign, and part of normal inter-individual variation. These need to be distinguished from those that are pathogenic. However, the pathological significance of genetic variants may be unclear (so-called variants of unknown significance (VUSs)). Such VUSs can be extremely frustrating for doctors and patients alike, as they do not assist with diagnosis or management, but can cause enormous anxiety and may trigger downstream testing, which may not assist with clarification. Laboratories may choose to report VUSs or not. The reporting needs to be balanced between reporting too little, which potentially risks missing a diagnosis, and reporting too many VUSs, leading to the potential for the mis- and overdiagnosis of patients, a factor that clinicians need to be aware of. As the field progresses, reporting VUSs may become more prominent by laboratories that wish to limit liability. However, as more patients have sequencing performed and knowledge increases, our ability to classify variants should improve, thus reducing the reporting of VUSs.

Interpretation of variants is complex and influenced by experience and an understanding of how mutations in a particular gene alter its function and cause disease. This could lead to the same variant being classified differently in different laboratories. Interpretations also change with time, as knowledge accumulates. This raises the issue of the value of re-analysis of genetic data. It is unclear whose responsibility it should be to initiate re-analysis; there are also no guidelines as to how frequently it should be done. Data are being gathered very rapidly and interpretation of a variant may thus change with time.

\section{Unexpected findings from genetic testing}

As large amounts of genomic data may be analysed in a test in search of the causative genetic fault, other unexpected incidental or secondary findings may be identified. These may be unrelated to the original indication for testing, but are of medical value for patient care. The possibilities of such findings need to be raised with patients in advance of testing. However, difficulties may arise as to which 
findings should be conveyed to patients and when. For example, should information about adult-onset diseases be conveyed to children? Individuals may be frustrated by a test that does not provide an answer for their clinical problem, but raises additional medical concerns, which now have to be dealt with. It is estimated that $\sim 1 \%$ of broad NGS-based genetic tests may reveal a reportable incidental variant. Some guidelines regarding incidental findings do exist, although they are by no means standardised. One such example is the American College of Medical Genetics (ACMG) reportable/actionable list of 59 genes. These genes include those associated with inherited cancer syndromes, cardiomyopathy, familial hypercholesterolaemia and Marfan syndrome. It obligates clinicians/genetic counsellors to report pathogenic and likely pathogenic variants in these genes to patients. However, there is currently little consensus across different centres with regard to these practices. Others recommend that data should not be analysed for such findings.

If a laboratory chooses to actively search for secondary findings, this should be performed separately from the original analysis and only if prior informed consent has been obtained from the patient. If carrier status is identified in adults, some recommend that it should be reported, as it may impact on reproductive options. It may also cause anxiety, as testing the partner in a recessive condition may be time-consuming, expensive and not provide reassuring answers.

\section{Challenges of genetic testing in Africa}

Genetic testing in individuals with African ancestry currently raises particular challenges. Critical to determining pathogenicity of variants is to compare their occurrence in patients and unaffected individuals. The paucity of data from normal individuals in Africa and the extensive genetic diversity on the continent make the possibility of finding a VUS significantly higher. There is an urgent need for diagnostic laboratories to collaborate and establish national data resources to ensure storage of data and sharing of variants so that interpretation on the African continent can be optimised.

Furthermore, there is little epidemiological knowledge regarding genetic disease in Africa, which makes it difficult to know which conditions should be prioritised for testing. Research projects such as those funded by the Human Heredity and Health in Africa (H3Africa) initiatives are beginning to address these issues. Examples include the Deciphering Developmental Disorders in Africa (DDD-Africa) project, a joint venture between the University of the Witwatersrand, South Africa, and the University of Kinshasa, Democratic Republic of the Congo. Initiatives to study deafness and neurological disease in
Africa have also been initiated (https://h3africa.org/). These projects will ultimately lead to increased knowledge and more directed diagnostic approaches in Africa.

\section{Conclusion}

Genetic testing is increasingly available for a broad range of conditions. Choice of tests is complex. Interpretation of tests is highly reliant on sound clinical information and assessment, as well as skilled laboratory and genetic personnel. The most appropriate test should be chosen to optimise results and minimise uninterpretable or unwanted findings. The same results may be interpreted differently with time as knowledge increases. Many challenges remain, including technical, interpretative and ethical ones. Medical geneticists and genetic counsellors have extensive training in genetics, with detailed knowledge in clinical genetic assessment of patients, choice of tests and interpretation of results, and should be involved in such testing to ensure patients receive optimal care. Genetic testing promises to contribute enormously to improved patient diagnosis in the next few years, as our understanding of genetic disease expands and our ability to manage and interpret data improves.

Declaration. None.

Acknowledgements. None.

Author contributions. Sole author.

Funding. None.

Conflicts of interest. None.

\section{Recommended reading}

Burke W. Genetic testing. N Engl J Med 2002;347:1867-1875. https://doi.org/10.1056/NEJMoa012113 Hardwick SA, Deveson IW, Mercer TR. Reference standards for next-generation sequencing. Nature Rev Gene 2017;18(8):473-484. https://doi.org/10.1038/nrg.2017.44

Kalia SS, Adelman K, Bale SJ, et al. Recommendations for reporting of secondary findings in clinical exome and genome sequencing, 2016 update (ACMG SF v2.0): A policy statement of the American College of Medical Genetics and Genomics. Genet Med 2017;19(2):249-255. https://doi.org/10.1038/gim.2016.190 Matthijs G, Souche E, Alders M, et al. Guidelines for diagnostic next-generation sequencing. Eur J Hum Genet 2016;24(1):2-5. https://doi.org/10.1038/ejhg.2015.226

Miller DT, Adam MP, Aradhya S, et al. Consensus statement: Chromosomal microarray is a first-tier clinical diagnostic test for individuals with developmental disabilities or congenital anomalies. Am J Hum Genet 2010;86(5):749-764. https://doi.org/10.1016/..ajhg.2010.04.006

Severin F, Borry P, Cornel MC, et al. Points to consider for prioritizing clinical genetic testing severin $\mathrm{F}$, Borry P, Cornel MC, et al. Points to consider for prioritizing clinical genetic testing
services: A European consensus process oriented at accountability for reasonableness. Eur J Hum Genet services: A European consensus process oriented at acco

Vears DF, Sénécal K, Clarke AJ, et al. Points to consider for laboratories reporting results from diagnostic genomic sequencing. Eur J Hum Genet 2018;26(1):36-43. https://doi.org/10.1038/s41431-017-0043-9

Accepted 20 February 2019 